\title{
Role of Silver Diamine Fluoride in Dentistry
}

\author{
Zohaib Khurshid ${ }^{1, \Subset}$ Juzer Shabbir ${ }^{2} \quad$ Abdullah Khaled Al-Otaibi ${ }^{3} \quad$ Abdulelah Mohammad Alsalem ${ }^{3}$ \\ Abdulmalik Abdullah Alhamdan ${ }^{3}$ Rizwan Jouhar ${ }^{4} \quad$ Muhammad Adeel Ahmed ${ }^{4}$
}

\author{
${ }^{1}$ Department of Prosthodontics and Implantology, College of \\ Dentistry, King Faisal University, Al Ahsa, Kingdom of Saudi Arabia \\ 2Department of Operative Dentistry and Endodontics, \\ Liaquat College of Medicine and Dentistry, Karachi, Pakistan \\ ${ }^{3}$ College of Dentistry, King Faisal University, Al Ahsa, \\ Kingdom of Saudi Arabia \\ ${ }^{4}$ Department of Restorative Dentistry, College of Dentistry, \\ King Faisal University, Al-Ahsa, Kingdom of Saudi Arabia
}

\author{
Address for correspondence Zohaib Khurshid, BDS, MRes, \\ MDTFEd, FPFA, College of Dentistry, King Faisal University, \\ Al Ahsa 31982, Kingdom of Saudi Arabia \\ (e-mail: drzohaibkhurshid@gmail.com).
}
Abstract
Keywords
- dental caries
- silver
- fluoride
- staining
- arresting caries

Dental caries is the most prevalent disease in primary, mixed, and permanent dentition. Many preventive and treatment strategies were used in the last decades. Silver diamine fluoride (SDF) is a promising nontoxic material which proves to be a turning point concerning conservative dentistry if the patient and dental practitioners are adequately educated regarding it. Several advantages have been reported related to the use of SDF, including the procedure being pain-free and drill-free, patient- and doctor-friendly caries control strategy, and management of dentinal hypersensitivity.

\section{Introduction}

One of the primary reasons for the replacement or failure of the direct restoration in both primary and permanent dentition is secondary caries. ${ }^{1}$ Secondary caries is defined as carious lesion occurring in dental tissue at the interface between the restoration and the tooth. ${ }^{2}$ This problem can be attributed to high caries risk of the patient, ${ }^{3}$ the type restorative material used, ${ }^{3-5}$ and adhesive interface degradation in composite restoration. ${ }^{6}$ Strategies based on antimicrobial materials have been proposed for prevention of secondary caries, which includes antibacterial restorative materials, ${ }^{7}$ fluoridereleasing materials, ${ }^{7-9}$ chlorhexidine- and silver-releasing materials, ${ }^{10-12}$ silver diamine fluoride (SDF), ${ }^{13,14}$ fluoride rinses, and chlorohexidine and fluoride protective varnish. ${ }^{15-18}$ The fluoride varnish has a disadvantage of reducing the bond strength of composite to dentine. ${ }^{19}$

Silver diamine fluoride $\left(\mathrm{AgF}\left(\mathrm{NH}_{3}\right)_{2}\right)$ is a promising material having useful applications in clinical dentistry. It is a colorless, alkaline ( $\mathrm{pH} \sim 10$ ) agent which has been used for 50 years in dentistry. ${ }^{20-23}$ It is usually available in a $8-\mathrm{mL}$ bottle containing $38 \%$ SDF. One bottle of SDF can be used for the treatment of 125 sites. It has fluoride for remineralization, and silver as an antibacterial agent. This combination makes SDF a better prospect for prevention and control of caries compared with fluoride therapy like sodium fluoride varnish..$^{24}$ Ammonia is another element of SDF, which is responsible for keeping the constant solution level for a more extended period. ${ }^{25}$ SDF is considered as a safe and effective material, which, along with the potential to prevent dental caries, has a role in the inhibition of oral biofilm formation and treatment of hypersensitivity. ${ }^{25,26}$ SDF is a proven, cost-effective treatment modality. ${ }^{24}$ Animal studies related to SDF showed that histologically, there is no alteration of pulpal tissue in SDF-treated tooth, showing that SDF possesses minimal adverse effects..$^{27}$ The primary role of SDF is to prevent or treat caries conservatively.

In the 1960s, Japan approved the use of SDF as a therapeutic agent to arrest dental caries in children. ${ }^{25}$ Since then, SDF started gaining popularity in many other countries like Argentina, Brazil, China, Portugal, and Australia due to the cost-effectiveness and simple application. ${ }^{25}$ In 2014, Food and Drug Administration (FDA) cleared the first use of SDF to manage early childhood caries, prevent pits and fissure caries in erupting permanent molars, prevent root caries in the older people, sterilize infected root canals, and treat dentinal hypersensitivity (-Fig. 1). Being a painless modality, it is particularly indicated in noncooperative patients and children. 


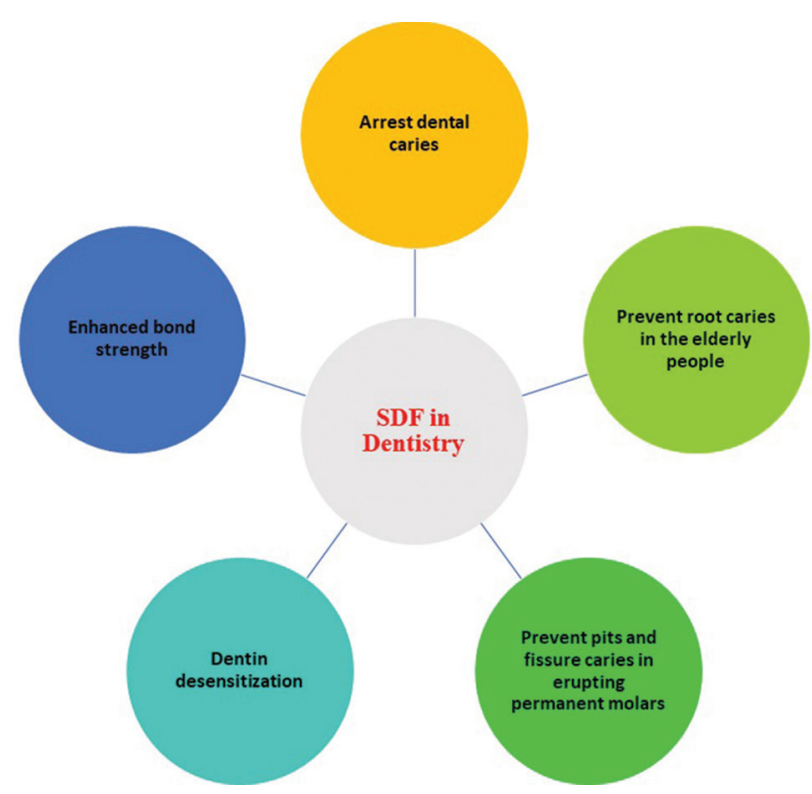

Fig. 1 Application of SDF in dentistry. SDF, silver diamine fluoride.

\section{Effect of SDF on Dental Caries}

Dental caries is the most common chronic dental disease affecting 60 to $90 \%$ of school-going children. In a recent study, dental caries was found to be shockingly prevalent, affecting $80 \%$ of the population of the Kingdom of Saudi Arabia. It is responsible for the loss of $50 \%$ of the missing teeth. In light of this problem, a global goal for 2020 was set up by World Health Organization (WHO) and Federation Dentaire Internationale (FDI) to guide the policymakers to help reduce the prevalence of caries and improve the oral health status of their population. Dental caries is a process of destructive chemical dissolution of dental hard tissues. This process is caused by the metabolism of dietary sugars and carbohydrates by the virulent bacteria, resulting in acid production and a decrease in $\mathrm{pH}$ of the biofilm covering the tooth surface. This acid results in loss of minerals from the surface of the tooth, resulting in the initiation of the carious lesion. Dental caries causes demineralization of enamel and dentine as well as degradation of type-I collagen of dentin. Dental caries, once developed, has adverse physical and psychological consequences such as pulp necrosis, cellulitis, distance infection, limitation of smiling and eating, and lower self-esteem and confidence.

Dental caries management in the last decade was focused on two main approaches-prevention, to limit the progress of caries at an early stage, and treatment, which includes drill and fills. The minimal invasive philosophy in caries management includes conservative management of caries with as little loss of dental hard tissue as possible. ${ }^{28}$ Strategy based on SDF has emerged as a technique which follows this philosophy. It can be used to arrest and prevent dental caries. The application of SDF on cavitated lesions increases the microhardness of dentin in a depth of $150 \mu \mathrm{m}$. This results in enhancement of the surface against the microorganisms. ${ }^{29,30}$

Moreover, treating demineralized enamel with SDF showed a lower overall mineral loss. ${ }^{31}$ Interestingly, it was demonstrated that when the enamel and dentin powder were mixed with SDF, there was a significant increase in the production of calcium fluoride and silver phosphate. ${ }^{32}$ Therefore, application of SDF on carious lesion results in the formation of a highly remineralized layer rich in calcium and phosphate content. Besides, inhibition of collagen type-I degradation was also observed. ${ }^{33-35}$ The matrix metalloproteinases (MMPs) and cysteine cathepsins possess an essential role in enzymatic degradation of collagen. The fluoride in SDF is proposed to have a potent inhibitory effect on the proteolytic enzymes, MMP-2, MMP-8, and MMP-9. ${ }^{34}$ A study performed by Hannah et al revealed that when MMP-9 was incubated with $150 \mathrm{ppm}$ of fluoride, a reduction of $79 \%$ was observed within a few minutes. ${ }^{36}$ The inhibitory effect of fluoride on MMP was shown to be due to the electronegativity of fluoride ions. ${ }^{37}$ SDF, when applied over a carious lesion, confirmed a significant colony-forming unit (CFU) reduction of Streptococcus mutans, Lactobacillus acidophilus, and Candida albicans. ${ }^{38}$ One study showed that SDF was able to reduce the number of $S$. mutans and Lactobacillus to a significantly low number within a week. ${ }^{39}$ Another study reported that the growth of cariogenic bacteria like S. Mutans, S. sobrinus, L. acidophilus, L. rhamnosus, A. naeslundii, and Staphylococcus aureus significantly decreased after the application of 20 ppm of silver nitrate solution. ${ }^{25,26}$

Today, the standards for the WHO goal appear to be achievable by using SDF as a caries preventive agent and a product to manage early childhood caries. ${ }^{25,40}$ American Academy of Pediatric Dentistry (AAPD) recently concluded that 38\% of SDF is currently the best evidence-based material to arrest caries for children, adults, and patients with special needs. ${ }^{41}$

\section{Mechanism of Action of Anticaries Activity}

The ability of silver to prevent caries is due to its positively charged ions which adhere to the bacterial cell wall and prevent bacterial aggregation. ${ }^{42}$ SDF has been hypothesized to exert its antibacterial action in several possible ways. First, the silver ions in SDF can interact with life-sustaining, crucial bacterial enzymes, and block the electron transport system. ${ }^{43}$ SDF can also communicate with the thiol group of the enzymes and cause enzyme deactivation, resulting in bacterial cell death. ${ }^{44}$ Second, the silver ions can bind electrostatically to anionic portions of the bacterial cell membrane or cell walls, resulting in inhibition of bacterial movement or cause membrane to leak or rupture. ${ }^{45}$ The silver compound is responsible for bacterial cytoplasmic extrusion because of the high reactivity of silver ions with phosphorous components and sulfur-containing proteins in the bacterial cell wall. ${ }^{26}$

Another mechanism by which SDF exerts its antibacterial action is through the interaction of its silver ions with bacterial deoxyribonucleic acid (DNA). Unless the DNA is contained within the nucleus, such as in eukaryotic cells, the interaction between the silver ions and bacterial DNA can result in the mutation of the DNA and bacterial cell death. ${ }^{44}$ Moreover, silver ions can prevent the bacterial aggregation process by electrostatically binding to phosphorus in DNA. ${ }^{26}$ Lastly, the silver ion can bind to amino acids of the bacteria to form an organometallic complex. 


\section{Effect of SDF on Dental Hypersensitivity}

Dental hypersensitivity is characterized by varying severity of pain, initiated by the thermal, chemical, tactile, evaporative, or osmotic stimulus. The most widely accepted theory of its pain mechanism is that the mentioned stimuli can cause inward or outward fluid displacement in dentinal tubules, which activates intrapulpal nerve endings. ${ }^{46,47}$ SDF has been shown to reduce the dentinal hypersensitivity in various studies. ${ }^{48-50}$ It prevents or treats dentinal hypersensitivity by occluding the dentinal tubules. The occlusion is achieved due to precipitation of proteins in dentinal tubules by silver ions. ${ }^{51}$ Moreover, fluoride ions in SDF can form calcium fluoride when these ions react with free calcium. The calcium fluoride can block the dentinal tubules. ${ }^{52}$

\section{Effect of SDF on Bond Strength of Restorative Materials}

Correct adhesion of filling materials to dentin is always desirable. However, the bond degradation is shown to occur with the time, which might result in caries and replacement of the restoration. ${ }^{13,53}$ The usage of fluoridated materials in restorative dentistry could potentially improve the longevity of the tooth-filling material and decrease the probability of secondary or "recurrent" caries. Thirty-eight percent of SDF can form a caries-resistant layer when applied to dentin. SDF has shown to increase dentin hardness because of the obturation of dentinal tubules with cariostatic silver ions. A cariostatic reaction between SDF and the adhesive restoration interface has been observed because of the local release of fluoride. The fluoride is responsible for remodeling of tooth minerals from hydroxyapatite to fluorapatite.

Furthermore, SDF prevents calcium and phosphate loss from dentin and inhibits MMP activity which ensures a durable adhesion between the filling and the dentin. ${ }^{54}$ Additionally, SDF facilitates the reparative dentin formation and produces a bioactive link with adhesive restorations. ${ }^{13}$ The major reactionary products resulting from the interaction of hydroxyapatite with SDF was found to be $\mathrm{CaF}_{2}$ and $\mathrm{Ag}_{3} \mathrm{PO}_{4}{ }^{55}$ It has been shown that when dentin treated with SDF was refreshed, the bonding strength of composite restoration was found to be highest as compared with just rinsing for 15 seconds. It was also shown that two-step self-etch adhesive and the universal adhesive used after phosphoric acid etching performed better when compared with universal adhesive alone on SDF-treated dental tissue. Additionally, SDF has been shown to perform better with the use of self-etch technique. ${ }^{56}$

Moreover, it was found that SDF + potassium iodide (KI) had no adverse effect on the bond strength of GIC to dentine and did not interfere with fluoride uptake into the demineralized dental tissue. ${ }^{57} \mathrm{SDF} / \mathrm{KI}$ combination has been shown to improve the microtensile and shear bonding strength of dentin with GIC significantly. ${ }^{13}$ On the other hand, a study reported contradictory findings..$^{53}$ However, the literature is tilted toward the opinion that SDF application does not result in an adverse effect on bond strength of dentin to adhesive restorations. ${ }^{56}$

\section{Drawbacks of SDF}

No adverse effect was reported with the use of SDF for 50 years in Japan. ${ }^{22}$ One clinical trial which included over 4,000 young children worldwide did not report any mortality or systemic toxicity by using the recommended amount of SDF. One significant local adverse effect is related to aesthetics. Permanent black stains of active carious lesions are the main disadvantage of SDF application. These stains can be seen clearly, especially if the tooth treated is anterior. ${ }^{58}$

For this reason, many patients refuse to be treated with SDF. A literature review regarding patients'/guardians' acceptance to permanent blackness of teeth after the treatment with SDF reported that $100 \%$ of parents were satisfied and accepted their children to be treated with SDF despite the aesthetic concerns. ${ }^{58}$ Contrarily, another study reported that only $26.9 \%$ of parents admitted treating their children with SDF for anterior teeth. ${ }^{58}$ To counter the undesirable staining effect, in 2005, a new approach was introduced. This approach suggested the application of saturated KI immediately after SDF placement. A recent study showed that at the time of SDF/KI protocol ( - Table 1) being used, there was an insignificant color variation when used under GIC as compared with irrigation with deionized water. The KI did not adversely affect bond strength. ${ }^{13}$

Recently, a commercial SDF/KI agent (Riva Star; SDI, Bayswater, Australia), consisting of 30 to 35\% SDF and a saturated solution of KI, was introduced for the treatment of hypersensitive dentine. Other local adverse effects include temporary stains of oral mucosa having "henna appearance" after its contact with oral mucosa. Since the dermis cannot absorb silver, the stains will disappear over $\sim 14$ days when keratocytes are shed. Permanent staining of the clothes or counters may also occur if the materials come in contact. Patients who are treated with SDF are advised not exceed

Table 1 Recommended protocol for potassium iodide ( $\mathrm{KI}$ ) staining reversal

Dispense an appropriate amount of SDF into disposable medicine cup (1 drop can be applied to at least 5 teeth with moderate-size cavities).

Apply petroleum jelly or use a rubber dam to protect soft tissue near affected areas.

Dry affected tooth surfaces as much as possible with air syringe or with cotton pellets.

Use a microbrush saturated with SDF to paint directly onto the tooth surface.

Avoid cavity margins or soft tissues.

Allow to absorb for 1 minute, then remove excess with cotton pellets.

Dispense an appropriate amount of $\mathrm{KI}$ into a disposable medicine cup.

Use a microbrush saturated with KI to paint directly onto the tooth surface, like SDF application. Reaction products form immediately.

Restore areas with resin-modified glass ionomer or composite restoration as indicated.

Source: Adapted from Garg et al. ${ }^{55}$

Abbreviation: SDF, silver diamine fluoride. 
$1.142 \mathrm{mg} \mid \mathrm{L}$ of drinking silver water. Moreover, SDF should not be used in patients allergic to silver. ${ }^{41}$

For stain reversal, the dosage of $\mathrm{KI}$ is comparable to $0.3 \mathrm{~mL}$ of saturated $\mathrm{KI}(1 \mathrm{~g} / \mathrm{mL})$ oral solution. When potassium iodide is applied, the reaction products are immediately formed and minute, safe amount of KI may reach the saliva. In the past, the synergistic effect of SDI and KI was investigated in the past for its impact on the permeability of demineralized dentin to S. mutans. ${ }^{57}$ However, only recently, the property of potassium iodide to treat staining was investigated. ${ }^{13,26,55,59}$ It is proposed that the discoloration inhibiting effect of potassium iodide does not adversely affect the caries inhibiting effect of SDF. The explanation suggested is there is a reaction of silver ions with iodide ions to form silver iodide. Also, the interaction of potassium iodide with carious dentin treated with SDF resulted in the formation of a white powder of tripotassium phosphate. This white powder is the major reason suggested for the black stain reversal. Another product formed is silver iodide. It is a photosensitive yellowish-white powder which can turn dark with exposure to light. The margins of restoration may still be at risk for discoloration. However, the overall staining is greatly reduced as compared with SDF alone. ${ }^{55}$ SDF and KI combination inhibited caries, but its efficacy was lower as compared with SDF alone. ${ }^{55}$ Therefore, the mixture should be used with careful case selection, such as anterior teeth. Another drawback is if the rubber dam isolation is not done or soft tissues are not protected with petroleum jelly, desquamative process such as ulcerative gingivitis may occur. However, these symptoms are temporary and subside within 48 hours. KI use should be restricted in pregnant and lactating women because KI may cause fetal harm, abnormal function of thyroid, and goiter. ${ }^{55}$ Data related to the effect of SDF and potassium iodide on pulpal tissue is scarce and requires further investigation.

\section{Conclusion}

SDF is a promising nontoxic material which can prove be a turning point concerning conservative dentistry if the patient and dental practitioners are adequately educated regarding it. Several advantages have been reported related to the use of silver diamine fluoride including the procedure being painfree and drill-free, patient- and doctor-friendly caries control strategy, and management of dentinal hypersensitivity. However, the main drawback of SDF is the staining of carious dentin. Therefore, case selection is an essential aspect of this method. One strategy suggested to counter this drawback is the use of potassium iodide in combination with SDF. Although SDF material seems highly biocompatible material, further research is needed to evaluate the molecular and histological long-term effects of SDF on dental hard and soft tissues before it can be recommended to be used routinely.

\section{Conflict of Interest}

None declared.

\section{References}

1 Kopperud SE, Tveit AB, Gaarden T, Sandvik L, Espelid I. Longevity of posterior dental restorations and reasons for failure. Eur J Oral Sci 2012;120(6):539-548

2 Hickel R, Peschke A, Tyas M, et al. FDI World Dental Federation: clinical criteria for the evaluation of direct and indirect restorations-update and clinical examples. Clin Oral Investig 2010;14(4):349-366

3 Opdam NJM, van de Sande FH, Bronkhorst E, et al. Longevity of posterior composite restorations: a systematic review and meta-analysis. J Dent Res 2014;93(10):943-949

4 Kuper NK, Opdam NJ, Bronkhorst EM, Huysmans MC. The influence of approximal restoration extension on the development of secondary caries. J Dent 2012;40(3):241-247

5 van de Sande FH, Opdam NJM, Truin GJ, et al. The influence of different restorative materials on secondary caries development in situ. J Dent 2014;42(9):1171-1177

6 Kuper NK, Opdam NJ, Bronkhorst EM, Ruben JL, Huysmans MC. Hydrodynamic flow through loading and in vitro secondary caries development. J Dent Res 2013;92(4):383-387

7 Ullah R, Zafar MS. Oral and dental delivery of fluoride: a review. Fluoride 2015;48:195-204

8 Zafar MS. Effects of surface pre-reacted glass particles on fluoride release of dental restorative materials. World Appl Sci J 2013;28:457-462

9 Ullah R, Zafar MS, Shahani N. Potential fluoride toxicity from oral medicaments: a review. Iran J Basic Med Sci 2017;20(8):841-848

10 Zhang JF, Wu R, Fan Y, et al. Antibacterial dental composites with chlorhexidine and mesoporous silica. J Dent Res 2014;93(12):1283-1289

11 Chatzistavrou X, Fenno JC, Faulk D, et al. Fabrication and characterization of bioactive and antibacterial composites for dental applications. Acta Biomater 2014;10(8):3723-3732

12 Cheng L, Weir MD, Xu HH, et al. Antibacterial amorphous calcium phosphate nanocomposites with a quaternary ammonium dimethacrylate and silver nanoparticles. Dent Mater 2012;28(5):561-572

13 Zhao IS, Mei ML, Burrow MF, Lo EC, Chu CH. Effect of silver diamine fluoride and potassium iodide treatment on secondary caries prevention and tooth discolouration in cervical glass ionomer cement restoration. Int J Mol Sci 2017;18(2):1-15

14 Horst JA, Heima M. Prevention of dental caries by silver diamine fluoride. Compend Contin Educ Dent 2019;40(3):158-163

15 Slot DE, Vaandrager NC, Van Loveren C, Van Palenstein Helderman WH, Van der Weijden GA. The effect of chlorhexidine varnish on root caries: a systematic review. Caries Res 2011;45(2):162-173

16 Autio-Gold J. The role of chlorhexidine in caries prevention. Oper Dent 2008;33(6):710-716

17 Tan HP, Lo ECM, Dyson JE, Luo Y, Corbet EF. A randomized trial on root caries prevention in elders. J Dent Res 2010;89(10):1086-1090

18 Affairs ADC; American Dental Association Council on Scientific Affairs. Professionally applied topical fluoride: evidence-based clinical recommendations. J Am Dent Assoc 2006;137(8):1151-1159

19 Leódido Gda R, Fernandes HO, Tonetto MR, Presoto CD, Bandéca MC, Firoozmand LM. Effect of fluoride solutions on the shear bond strength of orthodontic brackets. Braz Dent J 2012;23(6):698-702

20 Shah S, Bhaskar V, Venkatraghavan K, Choudhary P, Trivedi K. Silver diamine fluoride: a review and current applications. J. Adv. Oral Res 2014;5:25-35 
21 Mei ML, Nudelman F, Marzec B, et al. Formation of fluorohydroxyapatite with silver diamine fluoride. J Dent Res 2017;96(10):1122-1128

22 Yamaga R, Nishino M, Yoshida S, Yokomizo I. Diammine silver fluoride and its clinical application. J Osaka Univ Dent Sch 1972;12:1-20

23 Huang WT, Shahid S, Anderson P. Applications of silver diamine fluoride in management of dental caries. Advanced Dental Biomaterials. London, United Kingdom: Woodhead Publishing;2019:675-699

24 Rosenblatt A, Stamford TC, Niederman R. Silver diamine fluoride: a caries "silver-fluoride bullet" J Dent Res 2009;88(2):116-125

25 Zhao IS, Gao SS, Hiraishi N, et al. Mechanisms of silver diamine fluoride on arresting caries: a literature review. Int Dent J 2018;68(2):67-76

26 Hamama HH, Yiu CK, Burrow MF. Effect of silver diamine fluoride and potassium iodide on residual bacteria in dentinal tubules. Aust Dent J 2015;60(1):80-87

27 Rossi G, Squassi A, Mandalunis P, Kaplan A. Effect of silver diamine fluoride (SDF) on the dentin-pulp complex: ex vivo histological analysis on human primary teeth and rat molars. Acta Odontol Latinoam 2017;30(1):5-12

28 Zhao IS, Chu S, Yu OY, Mei ML, Chu CH, Lo ECM. Effect of silver diamine fluoride and potassium iodide on shear bond strength of glass ionomer cements to caries-affected dentine. Int Dent J 2019;69(5):341-347

29 Mei ML, Li QL, Chu CH, Lo ECM, Samaranayake LP. Antibacterial effects of silver diamine fluoride on multi-species cariogenic biofilm on caries. Ann Clin Microbiol Antimicrob 2013;12:4

$30 \mathrm{Chu} \mathrm{CH}$, Mei L, Seneviratne CJ, Lo EC, Lo M. Effects of silver diamine fluoride on dentine carious lesions induced by Streptococcus mutans and Actinomyces naeslundii biofilms. Int J Paediatr Dent 2012;22(1):2-10

31 Georgina S, Rosas P, Ángel M, Téllez A, Espinoza EV. In vitro efficiency of fluoride-containing compounds on remineralization of carious enamel lesions under cyclic $\mathrm{pH}$ conditions. Rev Odontol Mex 2014;18(2):96-104

32 Suzuki T, Nishida M, Sobue S, Moriwaki Y. Effects of diammine silver fluoride on tooth enamel. J Osaka Univ Dent Sch 1974; $14: 61-72$

33 Mei ML, Ito L, Cao Y, Lo EC, Li QL, Chu CH. An ex vivo study of arrested primary teeth caries with silver diamine fluoride therapy. J Dent 2014;42(4):395-402

34 Mei ML, Ito L, Cao Y, Li QL, Lo EC, Chu CH. Inhibitory effect of silver diamine fluoride on dentine demineralisation and collagen degradation. J Dent 2013;41(9):809-817

35 Mei ML, Chu $\mathrm{CH}$, Low KH, Che CM, Lo EC. Caries arresting effect of silver diamine fluoride on dentine carious lesion with S. mutans and L. acidophilus dual-species cariogenic biofilm. Med Oral Patol Oral Cir Bucal 2013;18(6):e824-e831

36 Hannas AR, Kato MT, Cardoso CdeA, et al. Preventive effect of toothpastes with MMP inhibitors on human dentine erosion and abrasion in vitro. J Appl Oral Sci 2016;24(1):61-66

37 Kato MT, Bolanho A, Zarella BL, Salo T, Tjäderhane L, Buzalaf MA. Sodium fluoride inhibits MMP-2 and MMP-9. J Dent Res 2014;93(1):74-77

38 Aas JA, Griffen AL, Dardis SR, et al. Bacteria of dental caries in primary and permanent teeth in children and young adults. J Clin Microbiol 2008;46(4):1407-1417

39 Koo H, Bowen WH. Candida albicans and Streptococcus mutans: a potential synergistic alliance to cause virulent tooth decay in children. Future Microbiol 2014;9(12):1295-1297

40 Duangthip D, Chen KJ, Gao SS, Lo EC, Chu CH. Managing early childhood caries with atraumatic restorative treatment and topical silver and fluoride agents. Int J Environ Res Public Health 2017;14(10):1-18

41 Crystal YO, Marghalani AA, Ureles SD, et al. Use of silver diamine fluoride for dental caries management in children and adolescents, including those with special health care needs. Pediatr Dent 2017;39(5):135-145

42 Morones JR, Elechiguerra JL, Camacho A, et al. The bactericidal effect of silver nanoparticles. Nanotechnology 2005;16(10):2346-2353

43 Marx DE, Barillo DJ. Silver in medicine: the basic science. Burns 2014;40(Suppl 1) :S9-S18

44 Russell AD, Hugo WB. Antimicrobial activity and action of silver. Prog Med Chem 1994;31:351-370

45 Slawson RM, Lee $\mathrm{H}$, Trevors JT. Bacterial interactions with silver. Biol Met 1990;3(3-4):151-154

46 Brännström $\mathrm{M}$, Aström $\mathrm{A}$. The hydrodynamics of the dentine; its possible relationship to dentinal pain. Int Dent 1972;22(2):219-227

47 Markowitz K, Pashley DH. Discovering new treatments for sensitive teeth: the long path from biology to therapy. J Oral Rehabil 2008;35(4):300-315

48 Craig GG, Knight GM, McIntyre JM. Clinical evaluation of diamine silver fluoride/potassium iodide as a dentine desensitizing agent. A pilot study. Aust Dent J 2012;57(3):308-311

49 Willershausen I, Schulte D, Azaripour A, Weyer V, Briseño B, Willershausen B. Penetration potential of a silver diamine fluoride solution on dentin surfaces. An ex vivo study. Clin Lab 2015;61(11):1695-1701

50 Castillo JL, Rivera S, Aparicio T, et al. The short-term effects of diammine silver fluoride on tooth sensitivity: a randomized controlled trial. J Dent Res 2011;90(2):203-208

51 Greenhill JD, Pashley DH. The effects of desensitizing agents on the hydraulic conductance of human dentin in vitro. J Dent Res 1981;60(3):686-698

52 Thrash WJ, Jones DL, Dodds WJ. Effect of a fluoride solution on dentinal hypersensitivity. Am J Dent 1992;5(6):299-302

53 Zhao IS, Chu S, Yu OY, et al. 1. Kucukyilmaz_et_al-2016-Lasers_in_Surgery_and_Medicine.pdf. J Conserv Dent 2018;37: 485-490

54 Lutgen P, Chan D, Sadr A. Effects of silver diammine fluoride on bond strength of adhesives to sound dentin. Dent Mater J 2018;37(6):1003-1009

55 Garg S, Sadr A, Chan D. Potassium iodide reversal of silver diamine fluoride staining: a case report. Oper Dent 2019;44(3):221-226

56 Yamaga M, Koide T, Hieda T. Adhesiveness of glass ionomer cement containing tannin-fluoride preparation (HY agent) to dentin-an evaluation of adding various ratios of HY agent and combination with application diammine silver fluoride. Dent Mater J 1993;12(1):36-44

57 Knight GM, McIntyre JM, Craig GG. Mulyani, Zilm PS, Gully NJ. An in vitro model to measure the effect of a silver fluoride and potassium iodide treatment on the permeability of demineralized dentine to Streptococcus mutans. Aust Dent J 2005;50(4):242-245

58 Magno MB, Silva LPD, Ferreira DM, Barja-Fidalgo F, FonsecaGonçalves A. Aesthetic perception, acceptability and satisfaction in the treatment of caries lesions with silver diamine fluoride: a scoping review. Int J Paediatr Dent 2019;29(3):257-266

59 Horst JA, Ellenikiotis H, Milgrom PL, Committee USCA. UCSF protocol for caries arrest using silver diamine fluoride: rationale, indications, and consent. J Calif Dent Assoc 2016;44(1):16-28 